Chinese Journal of Organic Chemistry

\title{
肝素类寡糖合成研究进展
}

\author{
尹晓娟 $a, b$ 间 俊 ${ }^{a, b}$ 姬胜利 $*, a, b$ 王风山*,a,b 曹鸿志*, \\ ( ${ }^{a}$ 山东大学国家糖工程技术研究中心 济南 250012) \\ ( ${ }^{b}$ 山东大学药学院 济南 250012)
}

\begin{abstract}
摘要 肝素(heparin, HP)和硫酸乙酰肝素(heparan sulfate, HS)是糖胺聚糖(glycosaminoglycans, GAGs)家族中的一类线性 硫酸化多糖，其结构复杂、生物活性多样. 为了深入研究其结构序列与不同生物活性之间的关系，近年来针对肝素类葟 糖的合成与功能研究成为糖化学和糖生物学研究的一个热点领域, 涌现出一些新的合成方法与合成策略. 选取了 2001 年以来国际上一些在肝素类寡糖的化学合成和酶法合成方面的代表性工作予以综述.
\end{abstract}

关键词 肝素; 硫酸乙酰肝素; 化学糖苷化; 糖基转移酶

\section{Recent Advances in the Synthesis of Heparan Sulfate Oligosaccharides}

\author{
Yin, Xiaojuan $^{a, b} \quad$ Yan, Jun $^{a, b} \quad \mathrm{Ji}$, Shengli ${ }^{*, a, b} \quad$ Wang, Fengshan ${ }^{*, a, b} \quad$ Cao, Hongzhi*,a \\ ( ${ }^{a}$ National Glycoengineering Research Center, Shandong University, Jinan 250012) \\ ( ${ }^{b}$ School of Pharmaceutical Science, Shandong University, Jinan 250012)
}

\begin{abstract}
Heparin (HP) and heparan sulfate (HS), a member of the glycosaminoglycan (GAG) family, are structurally related linear polyanionic polysaccharides. These complex sulfated polysaccharides can interact with hundreds of proteins and play essential roles in mediating or modulating a variety of physiological and pathological processes. Accumulated evidence has shown that the biological functions of HP and HS can be critically dependent upon their precise structure sequences. To decipher the sequence code of HS and HP, many research groups have focus on the development of novel strategies and synthetic methods for the assembling of heparin oligosaccharides during the last decade. The key developments since 2001 in the chemical or chemoenzymatic synthesis of heparin oligosaccharides are covered in this review.
\end{abstract}

Keywords heparin; heparan sulfate; chemical glycosylation; glycotransferase

肝素(heparin, HP)和硫酸乙酰肝素(heparan sulfate, HS)是由相同二糖重复单元通过 1,4-连接构成的线性硫 酸化多糖.二糖单元中葡糖胺 $(\mathrm{GlcN})$ 和糖酫酸之间的糖 苷键为 1,4-cis ( $\alpha$ 构型), 糖醛酸和葡萄糖胺之间的糖苷 键为 $1,4-\operatorname{trans}$ [对葡萄糖醛酸 (GlcUA) 为 $\beta$ 构型, 对 $L$-艾 杜糖醛酸 (IdoA)为 $\alpha$ 构型]. 对于 HP 来说其糖醛酸组成 $80 \% \sim 90 \%$ 为 $L$-IdoA, 每个二糖单元的平均硫酸化取代 度约为 $2.5 \sim 2.8$; 而 HS 中糖醛酸主要为 GlcUA, 二糖单 元的平均硫酸化取代度约为 0.8 (Scheme 1$)^{[1,2]}$.

$\mathrm{HP}$ 和 HS 的这种差异是一个宏观统计的结果, 事实 上 HP 多糖包含 HS 多糖上所有的寡糖序列, 反之亦然; 因而从寡糖序列的角度来说 HP 和 HS 是相同的, 只是一
些序列出现的频次不同而已. 为了避免混淆, 在本文中 将所有合成葟糖类型统称为肝素类葟糖. 由于 HP 或 HS 的糖醅酸可以为 GlcUA 或 $L-\mathrm{IdoA}, \mathrm{GlcN}$ 上的氨基可以 以硫酸化、乙酰化或未保护的氨基形式存在; 此外, $\mathrm{GlcN}$ 和糖醛酸上的各个羟基可以呈不同程度的硫酸化, 因而其结构是极其复杂的. 按照其生物合成途径推算, $\mathrm{HP}$ 和 HS 的潜在二糖重复单元有 48 个, 四糖重复单元 有 2304 个 $\left(48^{2}\right)$, 六糖重复单元有 110592 个 $\left(48^{3}\right)$. 这种 结构多样性赋予了 HP 和 HS 独特的生物活性多样性. 研 究表明 HP 和 HS 可以和数百种生长因子、趋化因子、 酶等蛋白质相互作用, 从而参与几乎所有的正常生理过 程以及炎症发生、病毒感染、肿瘤的发生与发展和神经

\footnotetext{
*E-mail: hzcao@sdu.edu.cn; fswang@sdu.edu.cn; shengliji@sdu.edu.cn

Received December 8, 2011; revised February 15, 2012; published online March 9, 2012.

Project supported by the National Natural Science Foundation of China (Nos. 20902087, 21172135) and the National Basic Research Program of China (973 program, No. 2012CB822102).

国家自然科学基金(Nos. 20902087, 21172135)和国家重点基础研究发展规划(973 计划, No. 2012CB822102)资助项目.
} 
退化性疾病等众多病理过程 ${ }^{[2,3]}$. HS 和 HP 与不同蛋白质 的选择性结合是与其精细的寡糖特定序列密切相关的, 包括该序列的糖醛酸类型和特有的硫酸化分布形式. 目 前研究最为深入并且结合序列得以确认的特定活性序 列有能与抗凝血酶 III (AT III)特异性结合的成糖序列 (Scheme 2, a); 能与成纤维细胞因子 II (FGF-2)特异性结 合的六糖序列 (Scheme 2, b) 和能与单纯性疮疹病毒 I (HSP-1)的 HSV gD 蛋白特异性结合的八糖序列(Scheme $2, \mathbf{c})^{[4]}$. 从猪小肠或牛肺中纯化得到的 HP 或 HS 多糖作 为抗凝剂在临床上已经应用了 50 余年. 20 世纪 80 年代, 由 Sinay, van Boeckel 和 Petitou 等 ${ }^{[5 \sim 7]}$ 完成的一系列肝素 类寡糖的合成为深入研究 AT-III 特异性结合肝素寡糖 片段提供了强有力的手段, 并最终促成了首个也是目前 唯一一个人工合成的肝素类寡糖抗凝药物——磺达肝 素(Fondaparinux, Arixtra ${ }^{\circledR}$, GSK)的上市. 磺达肝素与近 年来发展的低分子量肝素(LMWH)相比具有更好的抗 凝活性和更长的半衰期 $(15 \sim 10 \mathrm{~h})$, 同时能显著降低出 血风险等副作用, 且临床使用方便. 2006 年, 全球肝素 类药物的销售额达到 40 亿美元, 其中 LMWH 占 38 亿 美元. 磺达肝素自从 2002 年在欧美上市以来销售额逐 步上升, GSK 的年报显示, 其 2010 年销售额达到 4.8 亿 美元. 然而, 作为迄今合成步数最多(大于 50 步)的上市 药物, 磺达肝素也成为最贵的肝素类药物, 这极大的限 制了其更为广泛的应用.

除抗凝活性外, HP 和 HS 还具有抗炎、抑制或阻断 病毒感染和病原体入侵, 抑制新生血管生成和肿瘤的发 生等各种重要生物活性，因而发展肝素类抗炎、抗病毒、 抗肿瘤等药物成为近年来糖药物研究中的一个热点 ${ }^{[8,9]}$.

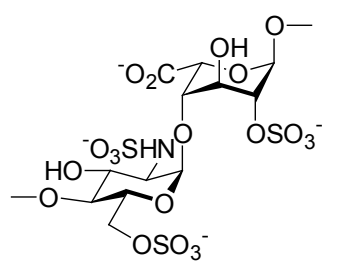

Major disaccharide unit of HP

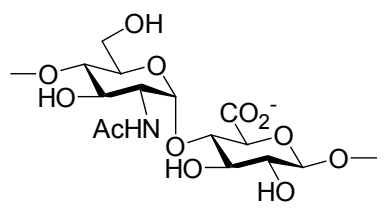

Major disaccharide unit of HS

\section{Scheme 1}

如前文所述, HS 或 HP 的各种活性是与其特定的寡糖序 列密切相关的, 要通过构效关系揭开其不同序列所蕴藏 的生物学信息就要求发展新方法、新手段来构建结构确 定的肝素类寡糖片段化合物库. HS 或 HP 的生物合成是 在高尔基体内通过复杂的多酶体系实现的. 首先, 是在 一个与核心蛋白相连的木糖-半乳糖-半乳糖-葡萄糖醛 酸的四糖序列非还原末端进行肝素二糖重复单元的合 成和糖链延伸. 这时肝素骨架的二糖重复单元为 $N$-乙 酰氨基葡萄糖(GlcNAc)和 GlcUA, 然后在一系列酶的加 工下将其转化为 HS 或 HP 多糖; 这些酶包括 $N$-去乙酰 化酶 $/ N$-磺酸基转移酶(NDST), 其可以将 GlcNAc 单元 上的 $N$-乙酰基脱除并对裸露出来的氨基进行硫酸化修 饰; 然后 C-5-位差向异构酶(C-5-epimerase, C-5-Epi), 通 过对 GlcUA 的 C-5-位构型翻转将其转化为 L-IdoA; 2位 $O$-磺酸基转移酶(2-OST)对 IdoA 的 C-2-位羟基进行 硫酸化; 3-位和 6-位 $O$-磺酸基转移酶(3-OST 和 6-OST), 催化在匍萄糖胺单元的 C-3-位和 C-6-位羟基引入磺酸 基. 由于参与糖链修饰的酶有不同亚型(目前已确定的 如 NDST 有 4 种, 6-OST 有 3 种, 3-OST 有 7 种, 2-OST 和 C5-Epi 各有 1 种)和底物适应性, 而且各步催化反应

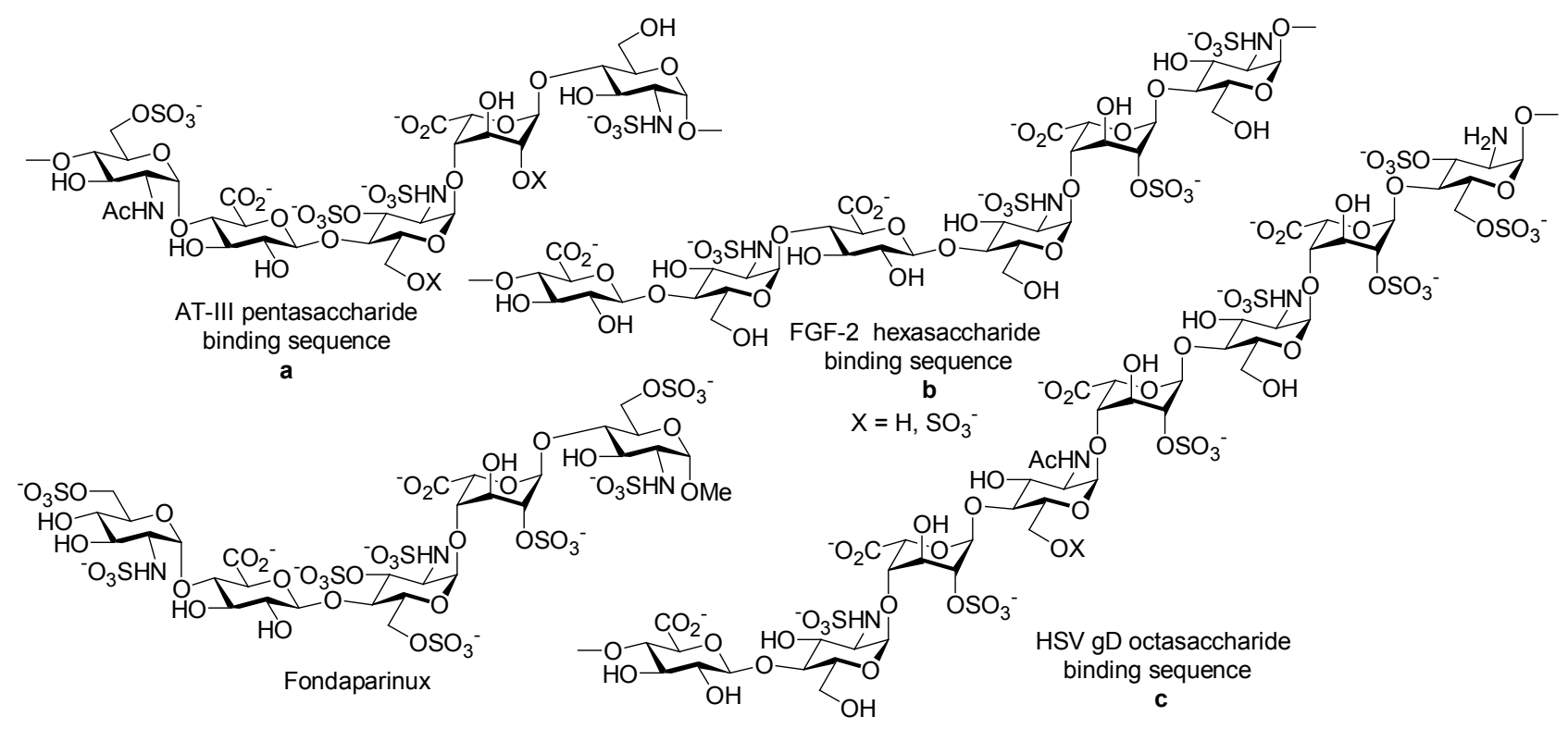

Scheme 2 
不能完全进行，因而，通过酶法合成途径获得结构均一 的肝素类葟糖面临巨大挑战.

由于肝素类寡糖的结构复杂性, 其化学合成一直以 来都是有机合成中最具挑战性的研究领域之一. 肝素类 寡糖的合成面临的主要困难有：(1)保护基的选择. 由于 肝素类寡糖的结构复杂性, 其众多羟基具有不同的硫酸 化分布形式，同时氨基可以为未保护氨基或被硫酸化、 乙酰化修饰, 因而要区分这些不同的羟基和氨基, 以便 于硫酸化时不相互干扰, 这就需要对保护基的选择进行 仔细的分析, 同时还要兼顾糖链延伸时糖基化位点上羟 基保护基的选择，保护基对给体和受体反应活性的影响 以及糖苷键形成时是否需要导向的邻基参与基团等因 素. 目前常见的保护基策略为运用酰基保护需要硫酸化 位点的羟基, 而苄基则被用来保护最终需要裸露的羟 基，其它位置上的正交保护基团在不同的合成策略中则 各不相同. (2) GlcN 和糖醛酸之间 1,2-cis-糖苷键 ( $\alpha$ 构型) 的立体选择性构建问题. 由于缺乏邻基参与作用, 2-脱 氧-2-氨基葡萄糖给体的 1,2-cis-糖苷化的立体选择性一 直以来是寡糖化学合成中的一个难点. 目前合成中通常 使用 2-位叠氮取代的葡萄糖基给体作为 $\mathrm{GlcN}$ 砌块的前 体, 叠氮糖作为 $\mathrm{GlcN}$ 前体省去了氨基上的保护基操作, 而且由于异头位效应(anomeric effect)糖苷化倾向于生 成 1,2-cis 的 $\alpha$ 糖苷键. (3) $L$-艾杜糖 ( $L$-Ido)或 $L$-IdoA 砌 块的制备难题. $L$-Ido 和 $L$-IdoA 砌块需要通过合成来制 备, 而之前的多步合成效率较低, 这也影响到肝素类寡 糖合成的总体效率. 此外, 多个位点上的同时硫酸化也 时常面临反应不完全等问题. 随着近年来新的寡糖合成 方法和合成策略的不断涌现, 国际上一些研究小组在肝 素类寡糖的化学合成上取得了一系列突破. 最近, 化学 酶法合成肝素类寡糖的成功则有望将酶法合成真正应 用于特定肝素萓糖序列的合成. 本文选取 2001 年以来 国际上一些代表性的工作对肝素类葟糖合成方面的最 新进展进行评述; 如需更全面的了解在此之前关于肝素 类葟糖合成方面进展 ${ }^{[7,10,11]}$ 及肝素类葟糖的生物合成途 径与受体分子的相互作用方面 ${ }^{[2,12]}$ 的综述请参阅所列的 代表性文献.

\section{1 化学合成肝素类寡糖}

\section{1 模块化合成}

\subsubsection{Seeberger 小组的合成}

立体选择性地构建 $\mathrm{GlcN}$ 与糖醛酸之间的 $\alpha 1-4$ 糖苷 键是肝素合成中的一个难点. Seeberger 等 ${ }^{[13]}$ 发现，具有 ${ }^{1} \mathrm{C}_{4}$ 构象的 $L$-IdoA 受体 2 在与 C-2-位叠氮取代的葡萄糖 三氯乙酰亚胺酯(TCI)给体 1 进行的糖苷化反应中能够 实现立体选择性的 1,2-cis 的 $\alpha$ 糖苷化; 然而具有 ${ }^{4} \mathrm{C}_{1}$ 构
象的 GlcUA 受体 5 在类似条件下则生成 $\alpha / \beta$ 混合物. 该 小组通过系统研究发现产物的立体选择性与 GlcUA 或 $L$-IdoA 的构象密切相关. 如 Scheme 3 所示, 糖醉酸受体 构象锁定为 ${ }^{1} \mathrm{C}_{4}$ 的受体(如 Scheme 3 中的 7,9)在一系列 的糖苷化反应中均立体选择性的生成 1,2-cis 的 $\alpha$ 糖苷 键，并且构象锁定为 ${ }^{1} \mathrm{C}_{4}$ 的受体具有更高的反应活性， 使得反应收率大大提高.

Seeberger 等 ${ }^{[14]}$ 运用此策略合成了一系列还原末端 为糖醀酸的二糖给体和受体模块, 期望将其应用于肝素 类寡糖的模块化合成. 然而, 具有不同序列的含糖醛酸 单元的给体和受体在三氯乙酰亚胺酯(TCI)作为离去基 团的糖苷化反应中显现出显著的差异. 例如, 二糖给体 11 与二糖受体 12 在促进剂 TMSOTf 存在下以 $88 \%$ 的收 率立体选择性地生成 1,2-trans 的 $\alpha$ 糖苷键(Scheme 4). 然而, 还原末端为糖醛酸 (GlcUA 或 L-IdoA)的三氯乙酰 亚胺酯糖基给体在一些糖苷化反应中未能得到正常糖 苷化产物(Scheme 4). 这表明给体和受体的糖链序列与 保护基的选择对于糖醛酸给体参与的糖苷化反应具有 极大的影响. 通过改变糖链序列, 运用 $[3+3]$ 策略, Seeberger 等 ${ }^{[14]}$ 成功的实现了肝素六糖骨架的组装并将其转 化为相应的硫酸化产物(Scheme 4).

尽管, Seeberger 等 ${ }^{[15]}$ 通过改变糖链序列实现了上述 肝素六糖的合成，但是其它一些还原末端为 $\mathrm{C} 2$-位叠氮 取代的葡萄糖基给体(如给体 19)在糖苷化过程中却给出 了 $\alpha / \beta$ 混合物. 如 Eq. 1 所示, 四糖给体 19 与二糖受体 20 之间的糖苷化以中等收率得到一对难于分离的 $\alpha / \beta$ 混合物, 而反应条件的改变也未能明显改善反应的立体 选择性 ${ }^{[15]}$.

非天然 $L$-IdoA 单元的大量制备是肝素合成中的一 个制约因素, Seeberger 小组 ${ }^{[16]}$ 发展了一个从 $D$-木糖出 发合成 $L$-IdoA 和 GlcUA 砌块的新途径. 如 Scheme 5 所 示, 木糖经 8 步反应转化为醛 $\mathbf{2 2}, \mathbf{2 2}$ 经 $\mathrm{MgBr}_{2}-\mathrm{OEt}_{2}$ 促进 的 Mukaiyama aldol 反应立体选择性的得到开链的葡萄 糖醛酸中间体 23. 中间体 23 再经 C-4-位羟基 Lev 保护, C-5-位 TBS 脱除, NIS-TFA 参与的脱除 C-1-位的硫乙基 缩醛保护并同时关环等三步反应得到 GlcUA 砌块 24. $L$-IdoA 砌块 $\mathbf{2 8}$ 可以通过醛 $\mathbf{2 5}$ 的立体选择性氰基加成以 及 Pinner 反应作为关键合成步骤得到. 尽管此合成路线 较长, 但是各步反应收率较好, 立体选择性专一, 可以 在克级规模上同时合成正交保护的 GlcUA 和 $L$-IdoA 砌 块, 因此, 其仍具有广泛的应用前景.

\subsection{Boons 小组的合成}

Boons 等 ${ }^{[17,18]}$ 发展了运用正交保护的二糖合成砌块 来构建肝素类寡糖的模块式合成策略. 如 Scheme 6 所 示, 在其保护基策略中, 需要硫酸化的羟基用 Lev 保护, 

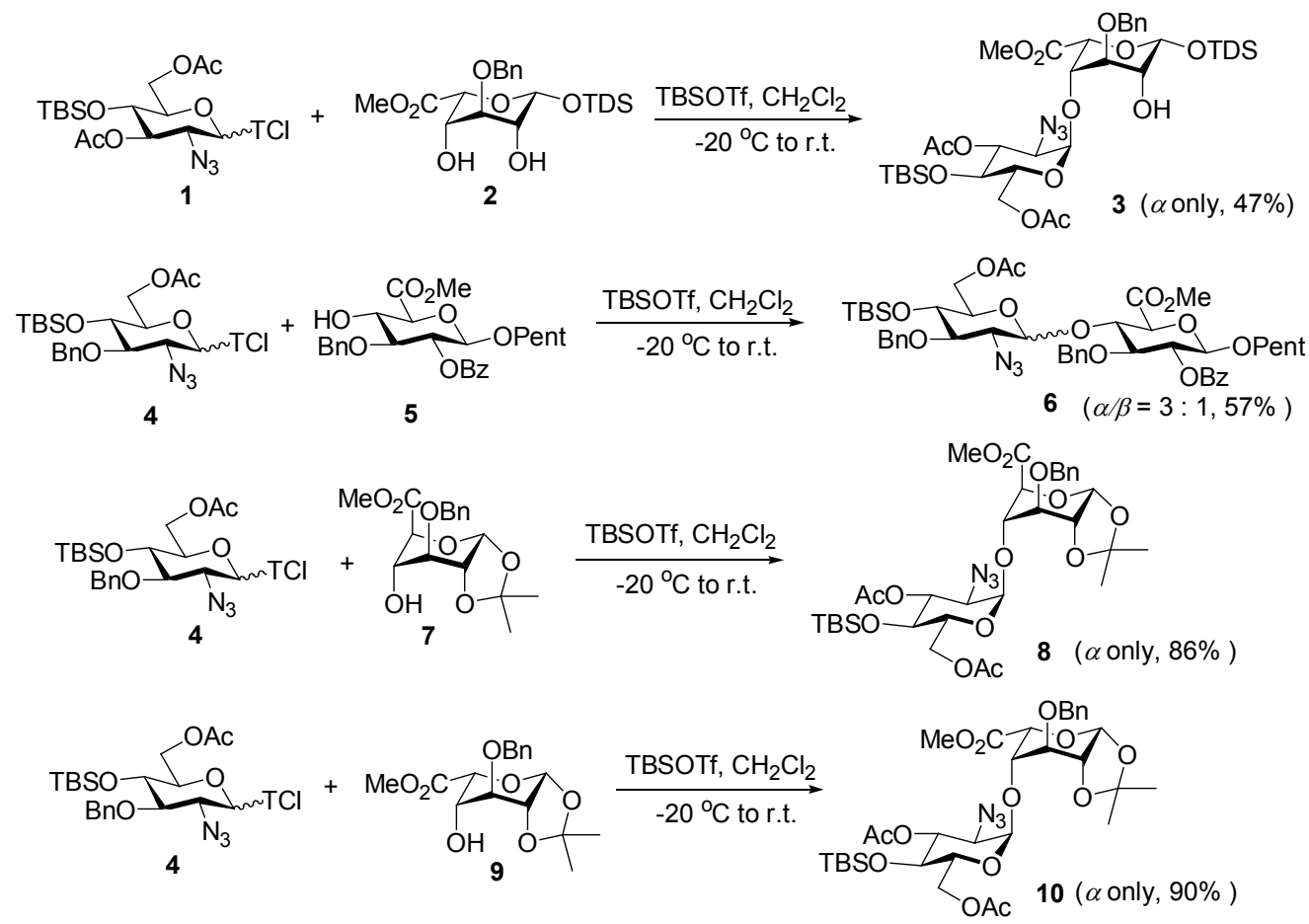

Scheme 3
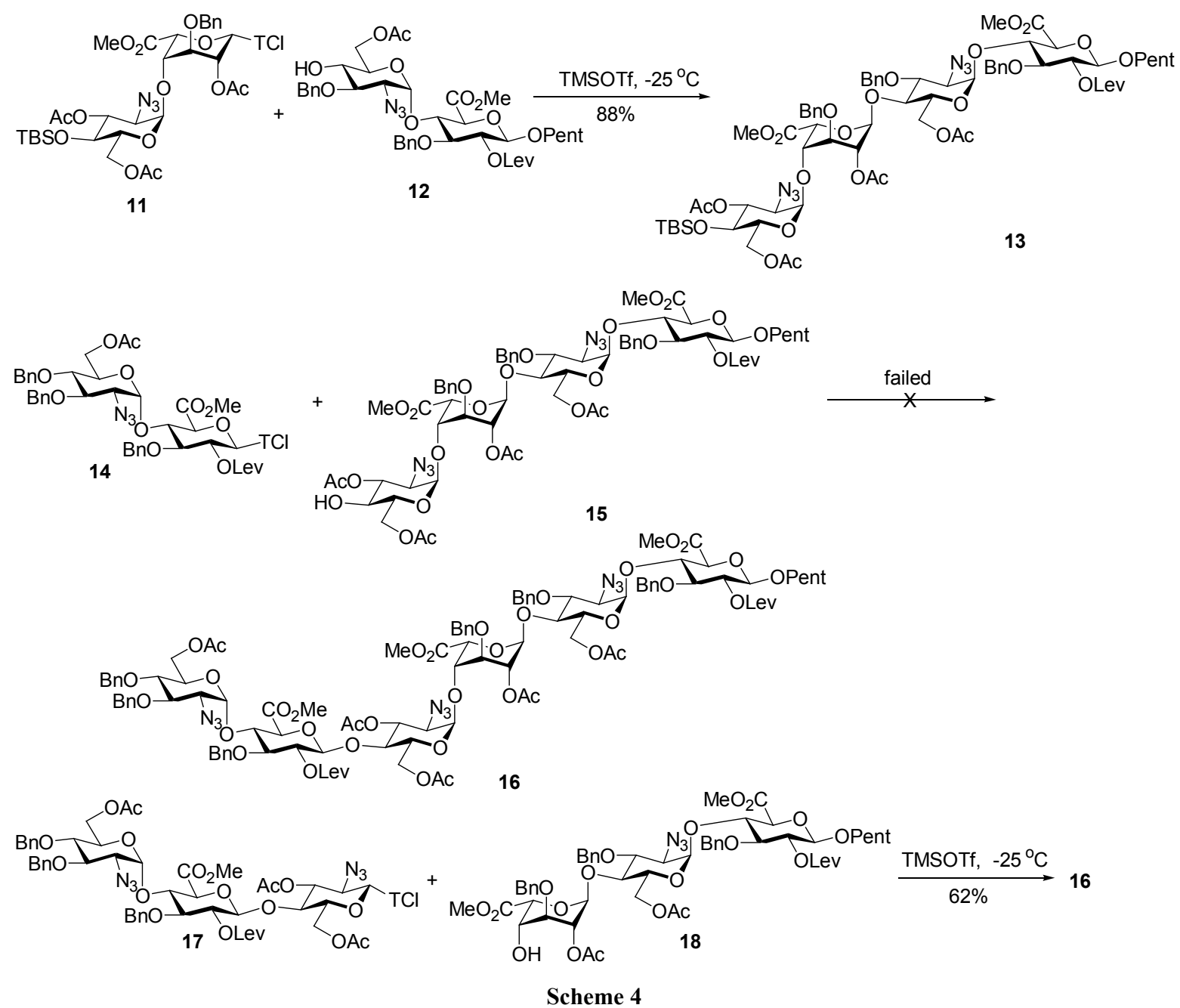

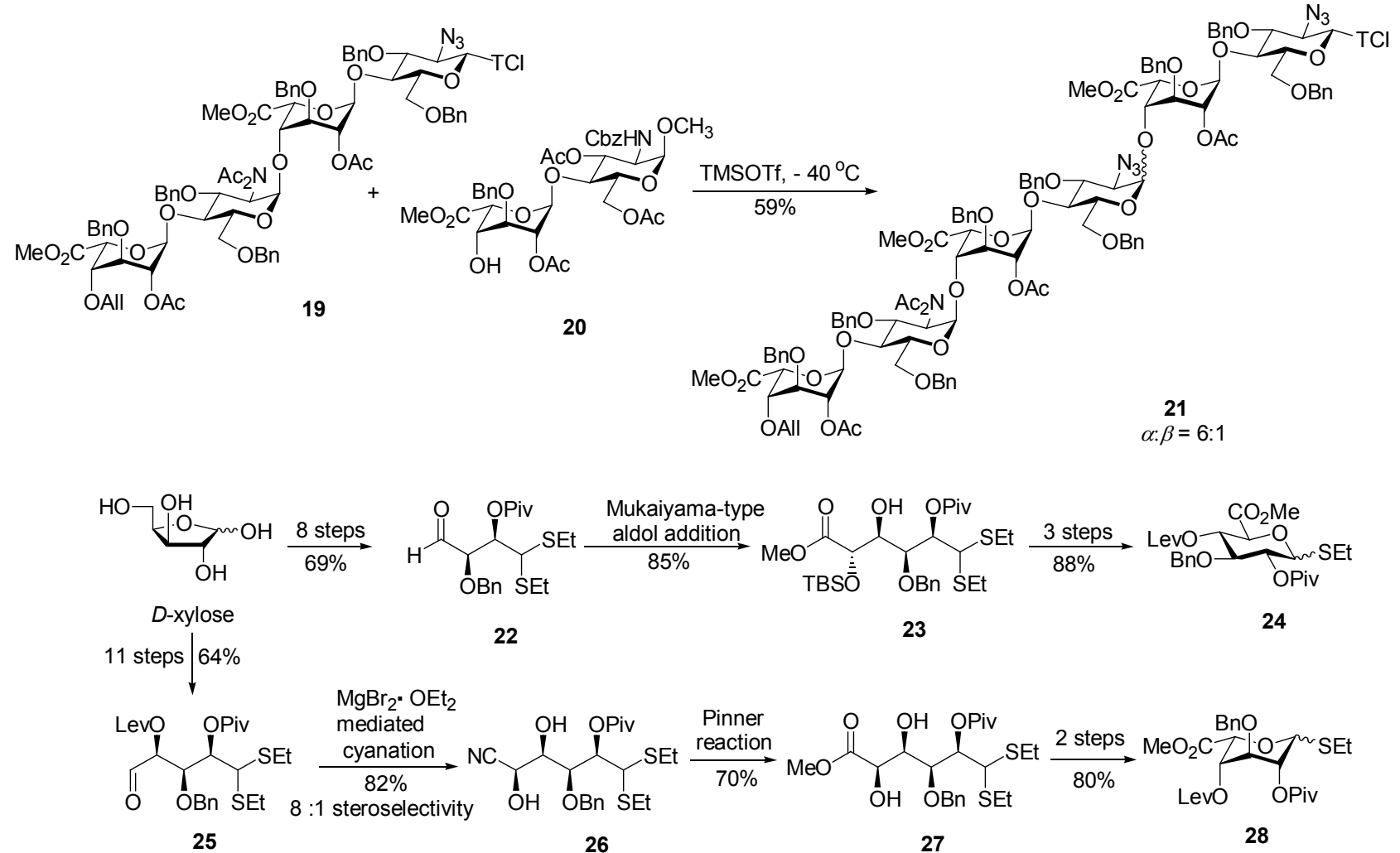

Scheme 5

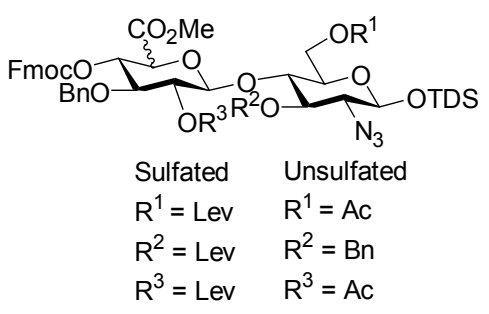

Scheme 6

不需要硫酸化的羟基用 $\mathrm{Ac}$ 和芐基保护, Ac 作为邻基参 与基团用以立体选择性的构建二糖模块中的 1,2-trans糖苷键. 二糖模块中糖链延伸位点上的 C-4-位羟基用 Fmoc 保护, 这样可以借鉴肽链固相合成中的 Fmoc 策 略, 有望实现脱 Fmoc 保护和随后的糖苷化反应 “一锅 法”完成.

Boons 等 ${ }^{[18]}$ 运用二糖模块成功的构建了一系列四糖 和六糖硫酸化肝素寡糖片段. 如 Scheme 7 所示, 二糖三 氯乙酰亚胺酯给体 $\mathbf{2 9}$ 与二糖受体 $\mathbf{3 0}$ 在 TMSOTf 存在下 立体选择性的生成四糖 32 . 选择性脱除四糖 32 非还原 末端 $L-I d o A$ 单元上的 Fmoc 保护基就得到四糖受体, 该 受体和二糖给体 31 进行第二个糖苷化反应就立体选择 的构建了肝素六糖骨架 33. 六糖骨架 33 经多个保护基 操作， $O$-硫酸化和 $N$-硫酸化等 9 步反应，以 $11 \%$ 收率得 到了硫酸化肝素六糖 34. 在 Boons 小组的合成策略中,
通过利用几个有限的正交保护的二糖给体和受体模块 可以立体选择性的构建一系列硫酸化肝素寡糖化合物 库. 如 Scheme 7 所示, 二糖给体 $\mathbf{3 1}$ 和二糖受体 $\mathbf{3 0}$ 可以 从相同的二糖模块得到.

\subsection{3 俞风䢠小组的合成}

近年来, 中国科学院上海有机化学研究所俞风选小 组 ${ }^{[19 ~ 23]}$ 对肝素类寡糖的化学合成开展了一系列研究工 作，发展了一个通过交替使用不同类型的糖基给体来实 现的模块式合成 ${ }^{[20]}$. 如 Scheme 8 所示, 二糖给体 35 和 $L$-IdoA 受体 36 在 TMSOTf 存在的条件下选择性的活化 三氯乙酰亚胺酯(TCI)给体 35 进而立体选择性的得到三 糖 37. 三糖 37 的苯硫基离去基团在上一步的糖苷化反 应中保持惰性，但是可以被 $\mathrm{BSP} / \mathrm{Tf}_{2} \mathrm{O}$ 促进剂体系活化， 通过与受体 38 偶联得到肝素四糖骨架 39. 用二糖给体 40 取代给体 35 , 在相同的反应条件下，该小组立体选择 性的构建了另外一个肝素四糖骨架 42. 俞入遂等通过模 块式合成完成了硫酸乙酰肝素酶(Heparanase)所能识别 的最小硫酸化肝素四糖底物的合成 ${ }^{[21]}$, 同时该小组运 用模块式合成完成了 Idraparinux 的合成 ${ }^{[23]}$.

Seeberger 小组, Boons 小组和俞风送小组等的模块式 合成只涉及氨基硫酸化的肝素序列的合成，而对于同时 含有 $N$-乙酰基, $N$-硫酸化和未取代氨基的相应肝素序列 的合成还有待于进一步研究. 


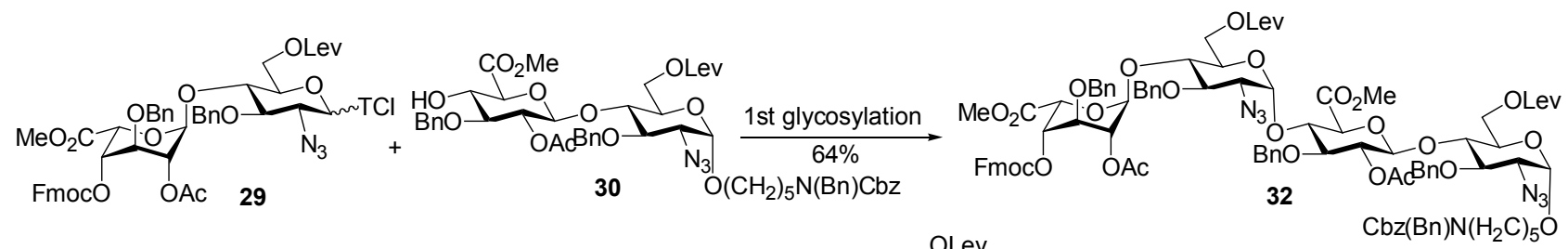

$\underset{\text { 1) deprotecting, } 82 \%}{\text { 2) } 31,2 \text { nd glycosylation, } 65 \%}$
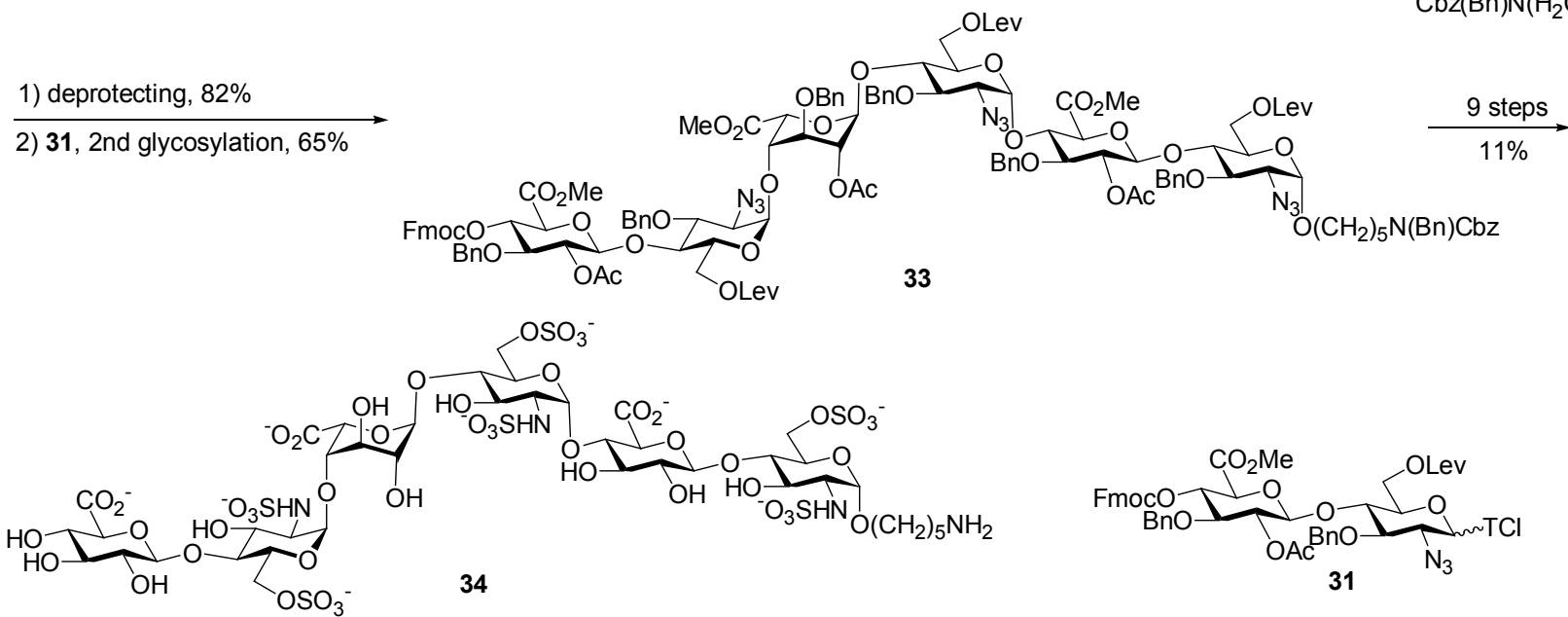

Scheme 7

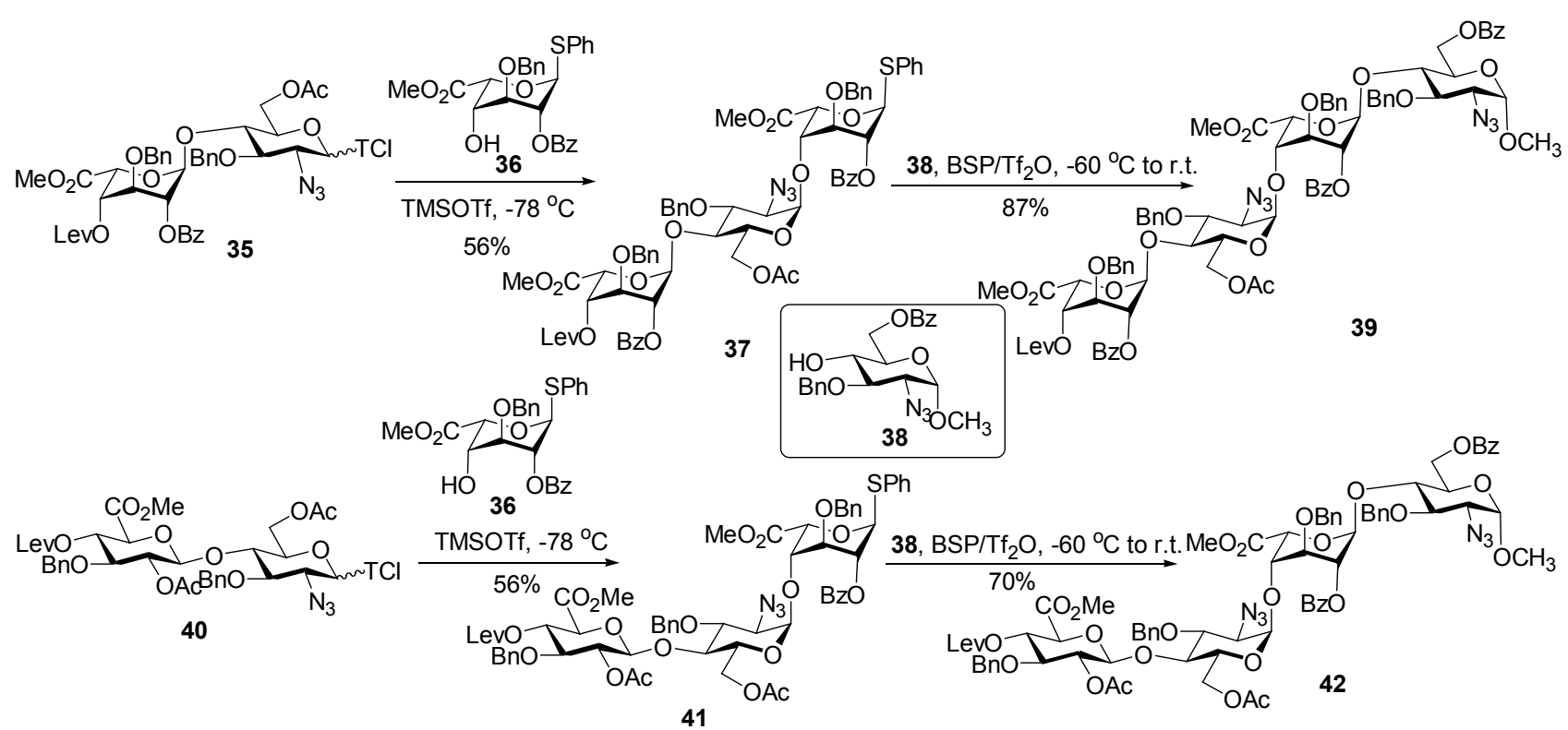

Scheme 8

\section{1 .4 洪上程研究组}

非天然 $L$-Ido 及其糖醛酸单元的获得一直是肝素类 葟糖合成中的一个制约因素, 洪上程等 ${ }^{[24]}$ 于 2001 年报 道了一个从葡萄糖中间体 $\mathbf{4 3}$ 出发两步合成 $L$-Ido 砌块 44 的新方法(Scheme 9). 苯甲酰基可以选择性的引入 $L$-Ido 砌块 44 的 C-2-位差基, 使得其可以方便的转化为 糖基受体用于构建肝素类葟糖.

洪上程等运用上述方法获得的 $L$-Ido 中间体 44 合成

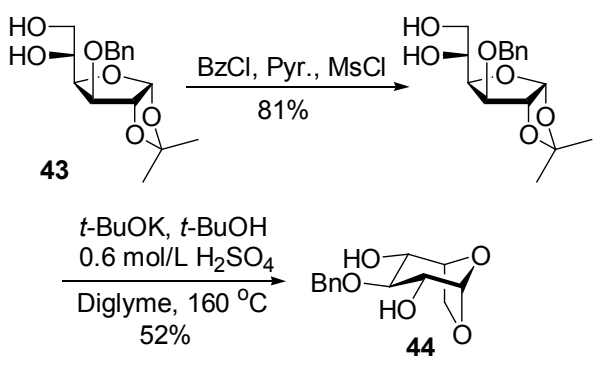
了二糖给体 45, 并将其作为唯一的糖基给体用于后续

Scheme 9 
的糖链延伸过程, 进而制备了含有 3 9 个糖单元的肝 素寡糖前体 47 50. 中间体 47 50 经脱除 $L$-Ido 单元 C-6-位 Lev 保护, TEMPO 参与的后期氧化将 $L$-Ido 单元 转化为 $L-\mathrm{IdoA}$; 再经过酰基脱保护, $O$-硫酸化和叠氮还 原， $N$-硫酸化等转化为硫酸化的肝素类寡糖 $51 \sim 54$ (Scheme 10) ${ }^{[25]}$. 运用此 $L$-Ido 中间体 44, 洪上程小组 ${ }^{[26]}$ 近来完成了所有 48 个肝素二糖片段的合成.

如前文所述, HSP-1 能特异性结合一个非常规肝素 八糖序列 (Scheme 2, c), 此八糖序列中的糖醛酸均为 $L$-IdoA, GlcN 同时存在 $N$-硫酸化、 $N$-乙酰化, 未保护的 氨基以及一个特定的 C-3-位 $O$-硫酸化基团. 最近, 洪上 程小组 ${ }^{[8]}$ 运用两个单糖模块 55, 56 和 3 个二糖模块 $57 \sim$ 59 完成了能与 HSP-1 的 HSV gD 蛋白特异性结合的肝 素类八糖 63 的合成(Scheme 11). 该合成先通过一个 $[1+2]$ 策略构建三糖给体 62, [1+2+2]策略构建戊糖受 体 61 , 三糖给体 62 和戍糖受体 61 通过 $[3+5]$ 组装出肝
素八糖骨架. 该八糖中间体经脱保护，后期氧化， $O$-硫 酸化, $N$-硫酸化等最终转化为硫酸化肝素八糖 63 . 合成 中所使用的 3 个二糖中间体 57 59 都可以从共同的二 糖中间体 60 制得, 60 中的 $L-I d o$ 单元的合成采用了上述 该小组自己发展的方法 ${ }^{[24]}$; 同时该小组也将其发展的 “一锅法”正交保护策略应用于单糖砌块 55 的制备中 ${ }^{[28]}$. 采用相似的策略, 洪上程等 ${ }^{[27]}$ 也合成了另外一个肝素 八糖序列，并将其与所合成的肝素八糖 17 以及市售的 肝素多糖一起用于抑制 HSP-1 感染实验. 在非洲绿猴肾 细胞(Vero cell)上进行的体外实验表明，肝素八糖 63 及 另外一个合成肝素八糖抗 HSP-1 感染的 $\mathrm{IC}_{50}$ 分别达到 5.4 和 $3.9 \mu \mathrm{g} \cdot \mathrm{mL}^{-1}$, 然而市售肝素多糖在 $100 \mu \mathrm{g} \cdot \mathrm{mL}^{-1}$ 水平上仍未能检测出明显的抑制作用. 此结果再次印证 了此前通过降解肝素片段所获取的 HSP-1 特异性结合 肝素八糖序列的确能抑制 HSP-1 的感染，为开发肝素类 寡糖抗 HSP-1 感染药物提供了新的思路.

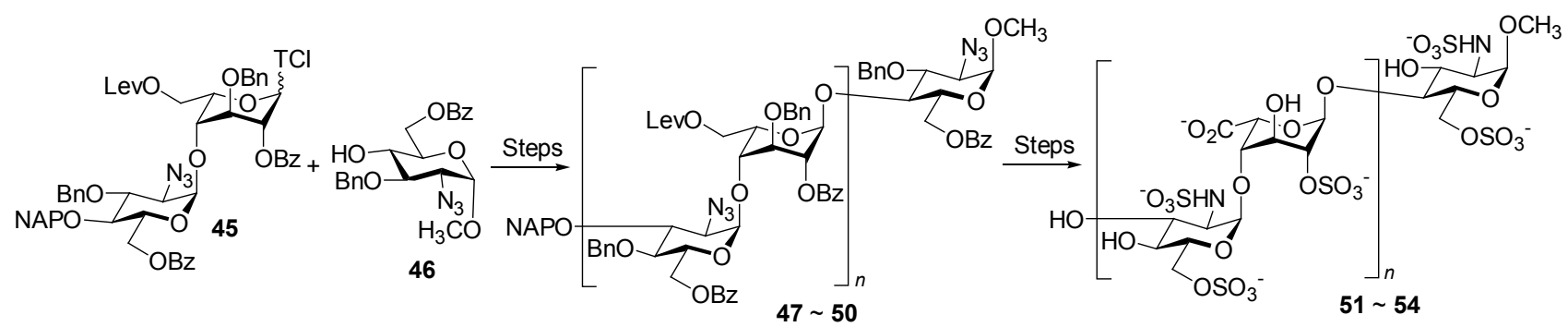

47, $n=1 ; \mathbf{4 8}, n=2 ; \mathbf{4 9}, n=3 ; \mathbf{5 0}, n=4$

$51, n=1 ; 52, n=2 ; 53, n=3 ; 54, n=4$

Scheme 10

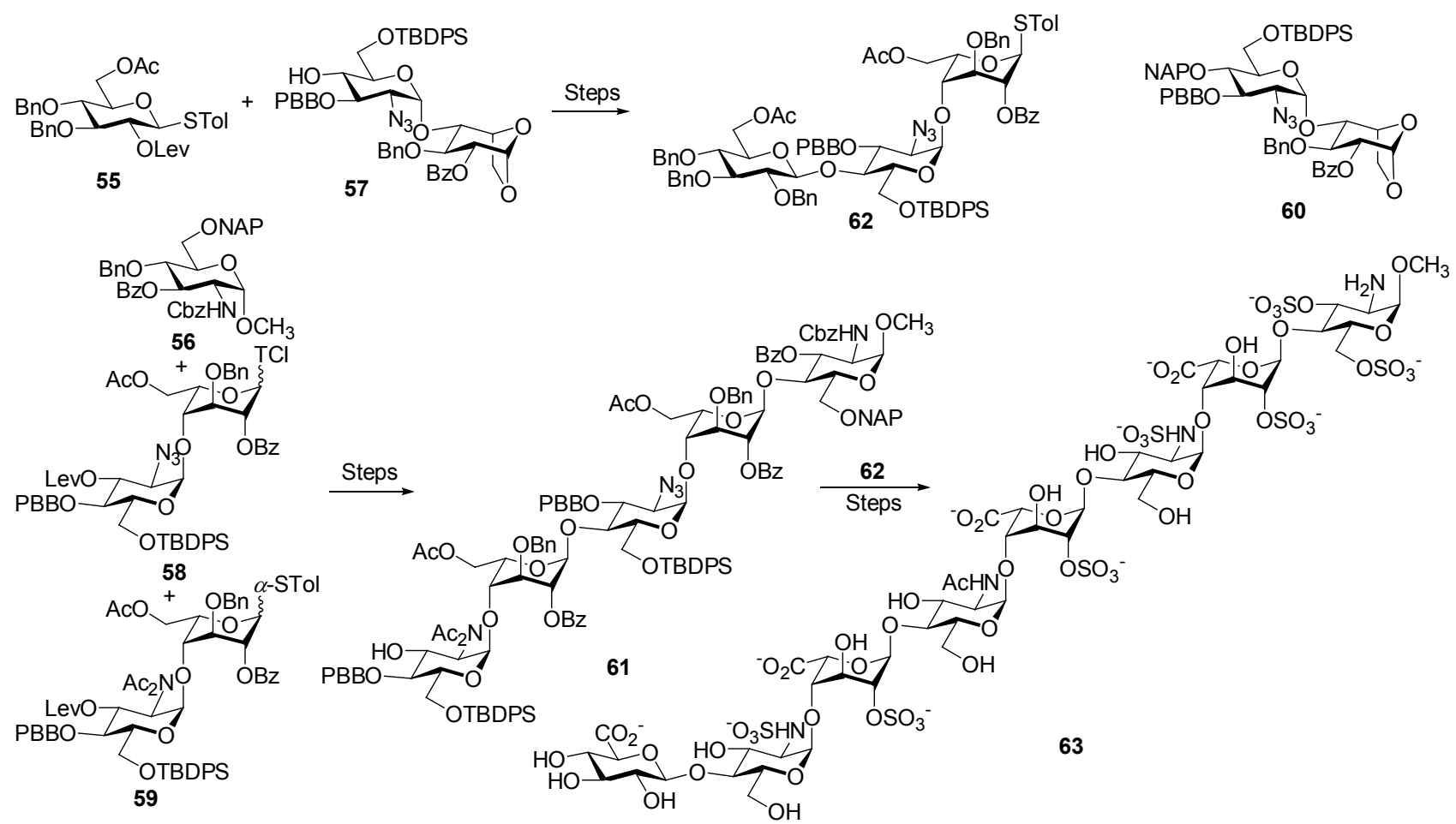

Scheme 11 


\subsection{5 van der Marel 小组的合成}

van der Marel 和 van Boom 等 ${ }^{[29]}$ 发展了一个运用单 糖砌块的连续糖苷化来组装肝素戊糖骨架的新策略. 该 合成首次将 $\mathrm{Ph}_{2} \mathrm{SO} / \mathrm{Tf}_{2} \mathrm{O}$ 介导的 $\mathrm{C}-1$-位羟基作为给体的 脱水糖苷化和 $\mathrm{BSP} / \mathrm{Tf}_{2} \mathrm{O}$ 促进的苯硫基给体的糖草化运 用于肝素类寡糖的合成. 由于 C-1-位羟基给体(如 64, 66)和苯硫基给体 65,68 可以分别在 $\mathrm{Ph}_{2} \mathrm{SO} / \mathrm{Tf}_{2} \mathrm{O}$ 和 $\mathrm{BSP} / \mathrm{Tf}_{2} \mathrm{O}$ 的不同条件下实现活化, 而且合成从非还原 端进行糖链延伸, 因而反应可以串联起来实现连续糖苷 化反应以高效构建肝素戊糖骨架 70 (Scheme 12). 与 Seeberger 等运用三氯乙酰亚胺酯给体在含有糖醛酸砌 块的合成中通常遇到的反应无法进行或立体选择性差 等问题不同, 脱水糖苷化和苯硫基糖苷给体在此类合成 中都显示出良好的反应活性和立体选择性. 尽管 van der Marel 和 van Boom 等的连续糖苷化反应需要中间体 的分离纯化, 不能实现真正意义上的 “一锅法” 合成, 但 是为将糖醛酸砌块直接应用于肝素类寡糖的合成提供 了一条新的解决途径.

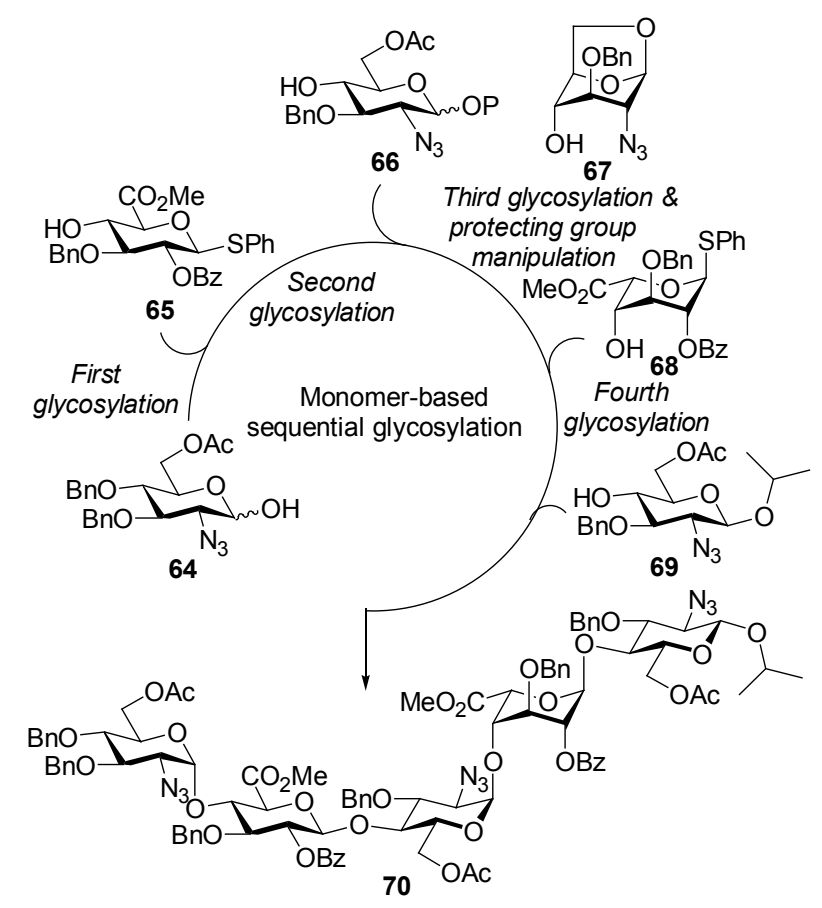

\section{Scheme 12}

\section{2 “一锅法” 合成}

\subsection{1 翁启惠小组}

翁启惠等近年来成功的将该小组早期发展的基于 糖基给体相对反应活性值(relative reactivity value, RRV) 而实现的 “一锅法” 寡糖合成策略 ${ }^{[30]}$ 应用于肝素类寡糖 的合成 ${ }^{[31]}$. 如 Scheme 13 所示, 由于单糖砌块 71 的相对 反应活性值为 53.7 , 二糖中间体 72 的 RRV 为 18.2, 因 而, 在仅有二者存在的情况下, 单糖砌块 71 作为给体被
选择性的优先活化, 而二糖中间体 72 则未被活化作为 受体用于构建三糖给体. 这样一个当场生成的三糖中间 体作为给体在后续加入的二糖受体 73 存在的情况下, 成功的通过 NIS-TfOH 的活化不经分离纯化, 以两步 20\%的总收率，“一锅法” 合成了肝素戊糖骨架 74. 74 再经过保护基操作, $O$-硫酸化, $N$-硫酸化等得到肝素成 糖 75. 该合成策略直接使用含有 GlcUA 和 $L$-IdoA 单元 的二糖砌块, 实现了这类低反应活性砌块在 “一锅法” 合成肝素类寡糖中的应用，为肝素寡糖的合成开辟了一 条新的途径. 然而, 由于不同合成砌块的 RRV 需要通过 反应去计算，反应总体收率偏低等，此合成策略在其它 肝素寡糖序列的合成中还面临众多挑战.
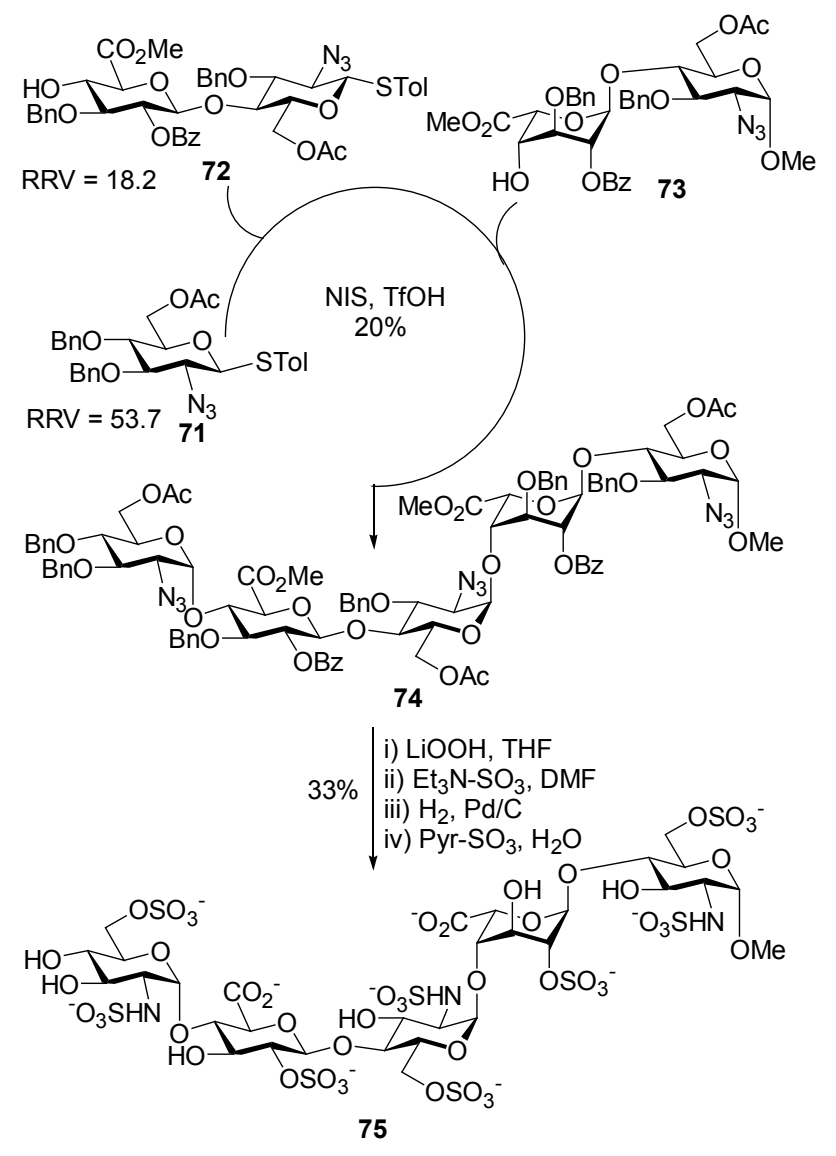

Scheme 13

此外, 翁启惠等 ${ }^{[32]}$ 还发展了一个通过自由基还原 异构化将 GlcUA 中间体转化为 $L$-IdoA 砌块的新方法. 如 Scheme 14 所示, GlcUA 中间体 76 经紫外光照射通过 自由基溴化在其 C-5-位引入溴，溴代中间体 77 经 $\mathrm{Bu}_{3} \mathrm{SnH}$ 引发的自由基还原异构化将 C-5-构型翻转, 从 而将 GlcUA 中间体 76 转化为 $L$-IdoA 中间体 $\mathbf{7 8}^{[32]}$.

\subsection{2 黄雪飞研究小组}

翁启惠等发展的 “一锅法” 肝素类寡糖合成依赖于 不同反应砌块间相对反应活性的差异, 来实现高反应活 性的砌块优先选择性的被活化. 但是, 相对反应活性 


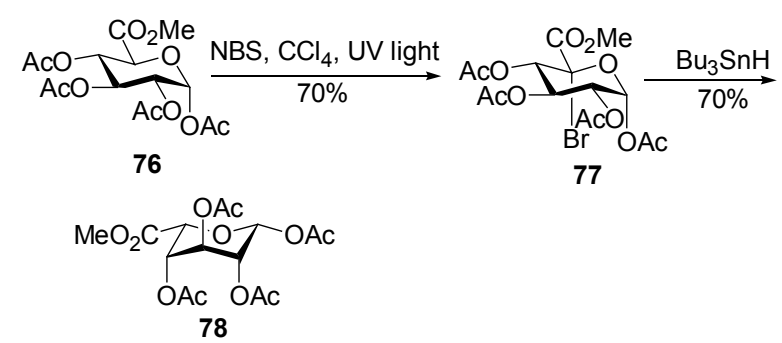

Scheme 14

值的测定需要大量的实验计算做基础, 因而应用上具有 极大的局限性. 近年来, 黄雪飞和叶新山等 ${ }^{[33]}$ 发展了一 个 “预活化” 策略实现了不依赖于砌块活性的 “一锅法” 连续臭糖合成. 最近, 黄雪飞小组 ${ }^{[34]}$ 将此策略成功应用 于组合化学式肝素类六糖化合物库的合成. 如 Scheme 15 所示, 二糖给体 79 在不存糖基受体的情况下被 1 equiv. 当场生成的对甲基苯硫基三氟甲磺酸酯 ( $p$-TolSOTf) 预先活化, 然后加入含有对甲苯硫基离去 基团的二糖受体 80, 这样预先活化的糖基给体 79 就和 后续加入的二糖受体 80 成功实现了偶联生成四糖中间 体. 而通过精心选择的 $p$-TolSOTf 活化剂在此过程中则 不会活化二糖受体 80 或新生成的四糖中间体. 通过升 高反应温度破坏掉过量的活化二糖给体 79, 降低温度, 加入二糖受体 81, 并加入另外一份 $p$-TolSCl 来生成新 的活化剂去活化体系中之前当场生成的四糖给体, 这样 就成功构建了肝素六糖骨架 82.

与翁启惠等发展的依赖于砌块相对反应活性的寡 糖 “一锅法” 合成相比，黄雪飞等的 “预活化一锅法” 合成避免了复杂的保护基操作来调控不同砌块的反应
活性，因而极大的提高了寡糖合成的效率.

\section{3 含有保护的磺酸基砌块在肝素类寡糖合成中应用}

在常规的肝素类寡糖合成中, 多个磺酸基团都是在 完成肝素骨架合成后，先选择性脱除需要硫酸化位点上 差基的酰基保护进行 $O$-硫酸化, 然后再通过催化氢化 脱除不需要硫酸化羟基上的茮基保护，同时将 GlcN 单 元上的叠氮还原为氨基再进行选择性 $N$-硫酸化. 肝素 类葟糖上多位点的硫酸化反应往往不能进行完全, 得到 结构相似, 难以分离的混合物, 这使得反应纯化周期长, 收率低, 极大的影响了合成的整体效率. 如洪上程小组 在硫酸化肝素七糖和九糖的合成中, $O$-硫酸化和 $N$-硫酸 化过程的总收率均低于 $10 \%$.

为了解决这一问题, 近年来, 人们发展了一些针对 磺酸基的保护基策略，期望通过使用预先硫酸化的合成 砌块来进行肝素寡糖分子的组装. 早期发展的如苯基、 乙基、三氟乙基、异丁基等磺酸基保护基由于脱保护反 应条件剧烈等不利因素仅限于二糖组装 ${ }^{[35,36]}$. Taylor 等 ${ }^{[37,38]}$ 发现三氯乙基保护的磺酸咪唑盐 84 可以用来在 糖链羟基高效的引入三氯乙基(TCE)保护的 $O$-磺酸基, 而且 TCE 可以在锌-甲酸铵的温和条件下脱除. 含有 $\mathrm{TCE}$ 保护的磺酸基糖基给体 87 与含有 $\mathrm{TCE}$ 保护的磺酸 基受体 88 在未经优化的糖苷化条件下以良好的收率得 到含有两个 $\mathrm{TCE}$ 保护的 $O$-磺酸基的二糖中间体 89 (Scheme 16). 二糖 89 中两个磺酸基上的 TCE 保护基在 锌-甲酸铵的条件下可以方便的脱除 ${ }^{[37,38]}$.

俞入送等 ${ }^{[22]}$ 将 $\mathrm{TCE}$ 作为 $N$-磺酸基的保护基引入到 $\mathrm{GlcN}$ 单元(如 91)上, 并考察了其三氯乙酰亚胺酯给体

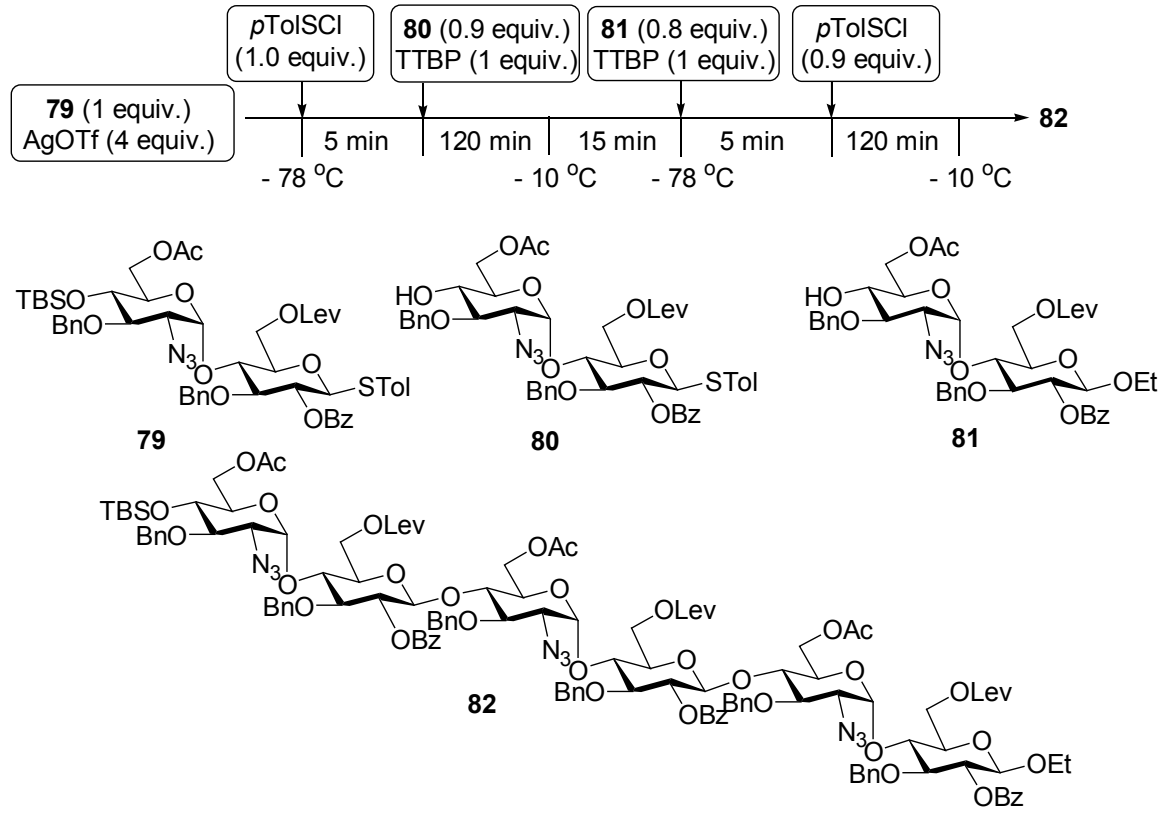

Scheme 15 


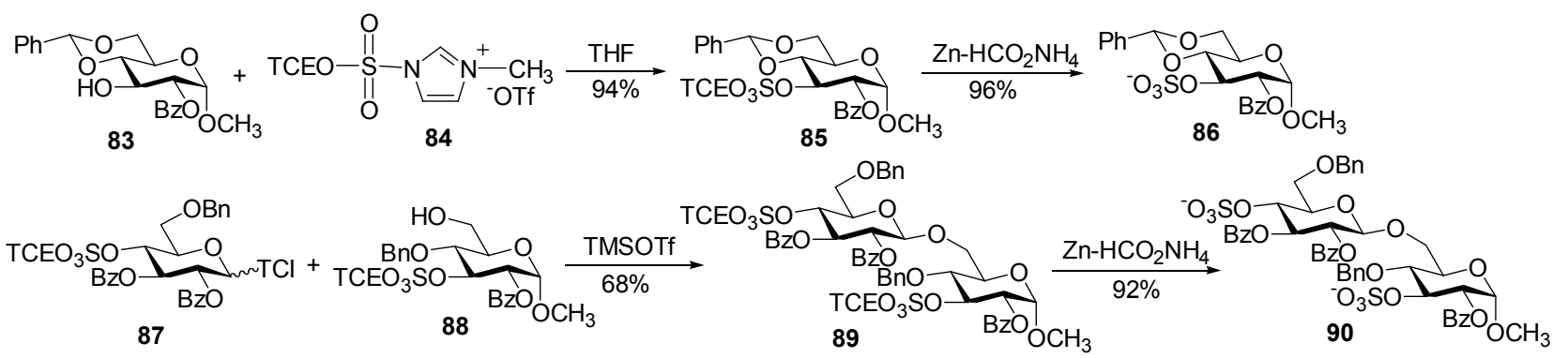

Scheme 16

的反应活性和脱保护方法. 如 Scheme 17 所示, TCE 保 护的磺酸基三氯乙酰亚胺酯给体 91 在促进剂 TMSOTf 存在的条件下立体选择性的生成 1,2-trans 的 $\beta$-糖苷键, 二糖 93 中磺酸基上的 TCE 保护基可以在锌-氯化铵的 甲醇溶液中高效的脱除.

从 Taylor 和俞风逖等的研究结果可以看出, TCE 是一 个很好的 $O$-磺酸基和 $N$-磺酸基保护基团, 其可以方便 的引入也可以在温和的反应条件下高效的脱除. 但是由 于 C-2-位 TCE 保护的 $N$-磺酸基给体在糖苷化中立体选 择性的生成 1,2-trans 的 $\beta$ 糖苷键, 因而 TCE 保护 $N$-磺 酸基给体不能适用于肝素类寡糖的合成. 最近黄雪飞 等 ${ }^{[39]}$ 将含有 TCE 保护的 $O$-磺酸基砌块成功的运用于硫 酸化肝素的合成. 如 Scheme 18 所示, 二糖给体与含有 $O$-磺酸基 TCE 保护的二糖受体 96 在促进剂 $p$-TolSOTf
存在条件下得到四糖中间体 97, 97 经脱除两个 C-6-位 Lev 保护，后期氧化，锌-甲酸铵的条件下脱除 TCE 保 护并同时将叠氮还原为氨基, $N$-硫酸化等 8 步反应转化 为硫酸化的肝素四糖 $\mathbf{9 8}^{[39]}$.

黄雪飞等工作表明，含有 $O$-磺酸基 TCE 保护的砌 块可以运用与肝素类寡糖的合成, 这类砌块能否应用于 更复杂的肝素寡糖骨架组装, 以及在复杂肝素骨架上对 多个 TCE 保护基的脱除是否高效, 尚有待于进一步的 考察.

\section{2 酶法与化学酶法合成肝素类寡糖}

由于 HS 或 HP 结构的复杂性，目前肝素类寡糖的化 学合成大多都是针对特定寡糖序列的定向合成. 其中涉 及到不同类型的多个保护基操作，因而反应步数多，合

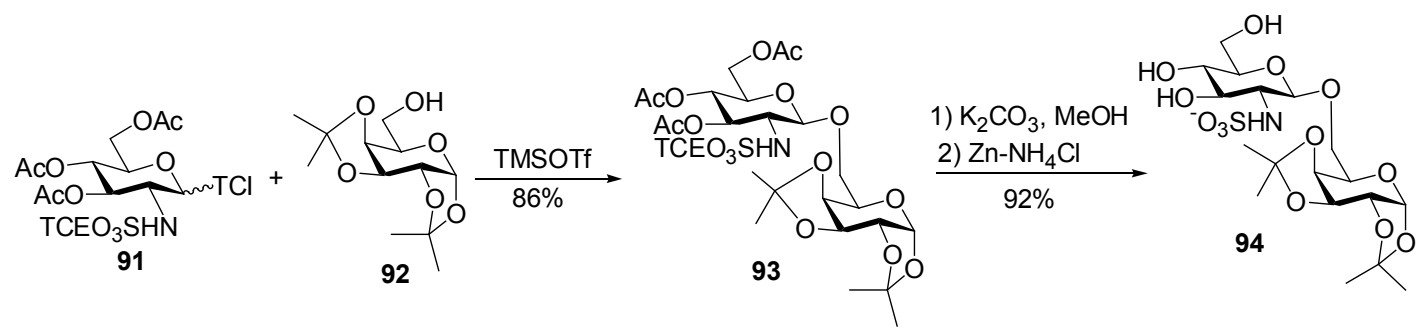

Scheme 17
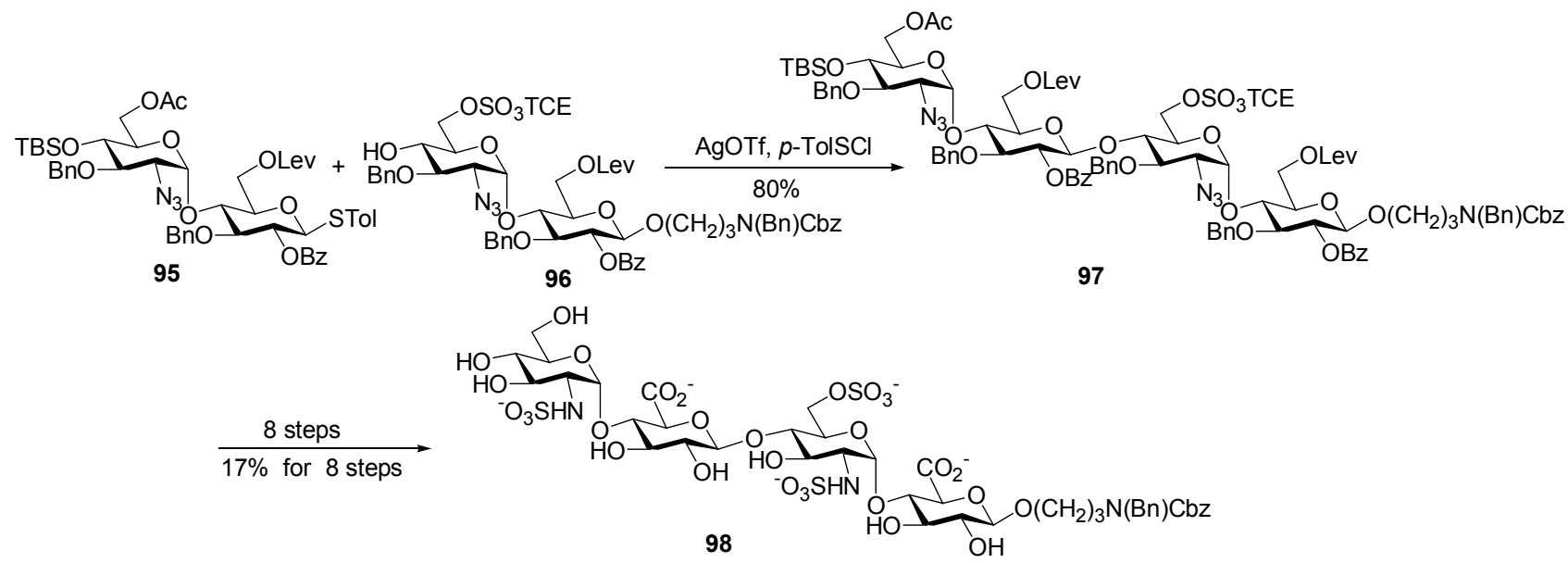

Scheme 18 
成效率低，对于不同序列的合成通常需要不同的合成砌 块与合成策略. 酶法合成由于无需保护基操作，立体选 择性专一, 因而合成效率高, 但是也面临酶的来源问题 以及底物适应性差等不足. 由于肝素的生物合成途径涉 及从肝素聚合酶、异构化酶和不同的磺酸基转移酶等多 个酶, 而且这些酶又有不同的亚型. 长期以来, 在肝素 的酶法合成研究一直集中在对不同来源的各个肝素合 成相关酶的催化机制和底物适应性方面. 一些运用肝素 多糖骨架, 如 $N$-和(或) $O$-脱硫酸化后的肝素多糖, 或肝 素合成前体(如 haparosan)等开展的酶法合成, 由于酶法 合成前体多糖结构复杂, 各步酶反应不能进行完全等, 因而得到的均为结构相似难以分离纯化的混合物.

刘建小组和 Linhardt 小组 ${ }^{[40,41]}$ 多年来一直从事肝素 的酶法合成方面研究, 最近他们小组合作发展了一个化
学酶法合成策略首次实现了肝素类寡糖的合成 ${ }^{[42,43]}$. 肝 素的生物合成途径是在一个与核心蛋白相连的四糖非 还原末端上开始骨架合成与糖链延伸的. 如 Scheme 19 所示, 刘建小组和 Linhardt 小组选取了一个可以从 haparosan 降解得到二糖 99 作为载体实现了肝素类寡糖 骨架的合成. 从 $E$ coli $\mathrm{K} 5$ 中得到的 $\mathrm{KfiA}$ 是一个 $N$-乙酰 氨基葡萄糖转移酶, 可以将 UDP-GlcNAc(或 UDPGlcNTFA)的 GlcNAc(或 GlcNTFA) 以 $\alpha 1-4$ 连接转移到糖 链非还原末端 GlcUA 单元的 C-4-位羟基上; PmHS2 可 以将 UDP-GlcUA 的 GlcUA 立体选择性的转移到糖链非 还原末端 GlcNAc(或 GlcNTFA)的 C-4-位羟基上. 刘建 和 Linhardt 等通过交替使用这两个酶实现了与二糖载体 相连的肝素戊糖骨架 103 的构建. 肝素戊糖骨架 103 中 同时含有 GlcNTFA 和 GlcNAc 单元, 其中 GlcNTFA 的

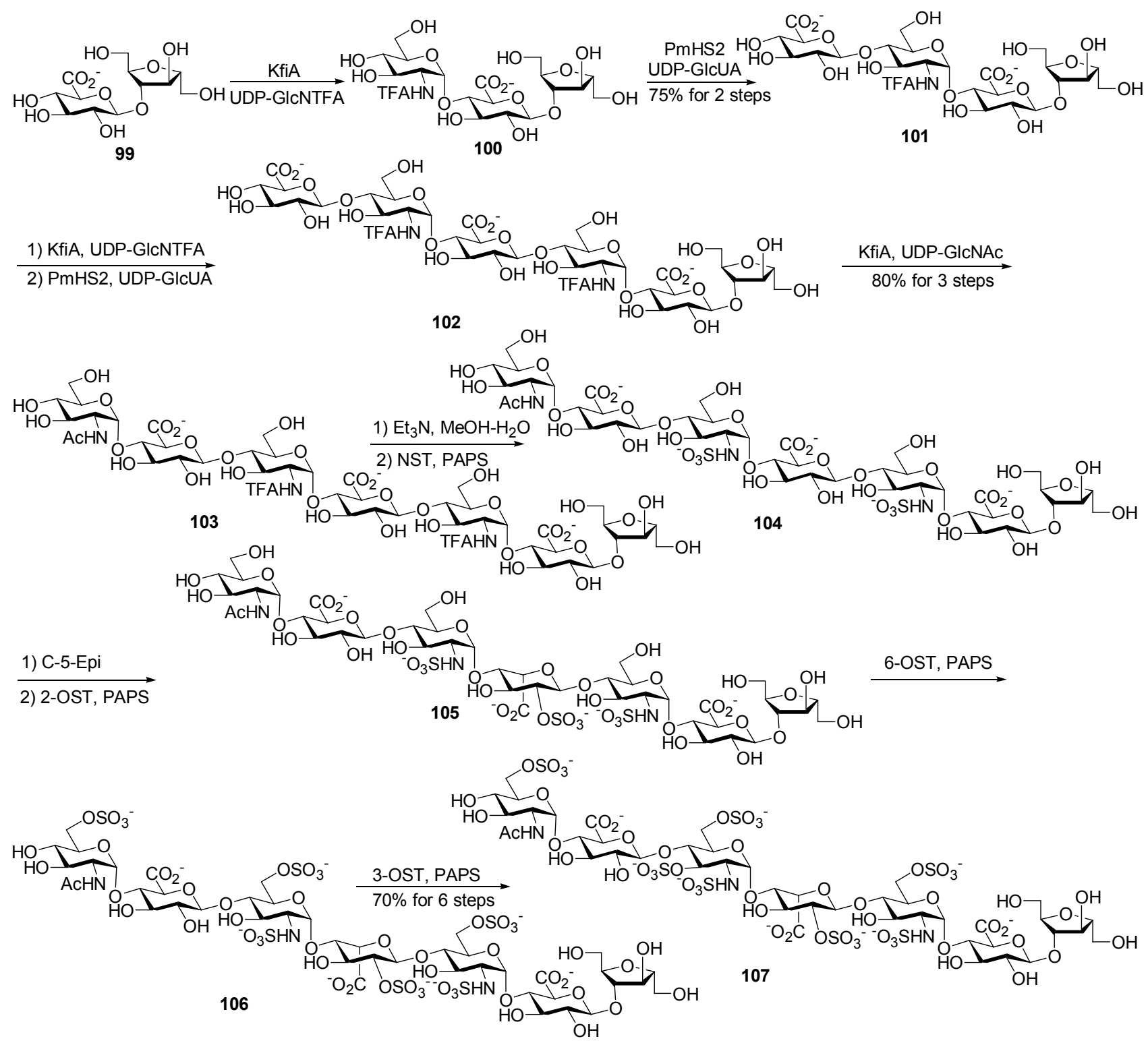

Scheme 19 
$N$-TFA 可以在碱性条件下方便的脱除, 裸露出的氨基进 而可以在 $N$-磺酰基转移酶 NST 催化下被硫酸化得到硫 酸化寡糖 104. 104 中非还原末端的 GlcNAc 单元可以确 保 C-5-Epi 只选择性的将还原端的 GlcUA 异构化为 $L$-IdoA, 2-OST 选择性的将 $L$-IdoA 的 C-2-位羟基硫酸化 得到 $N$-硫酸化 $L$-IdoA 2-位硫酸化的七糖中间体 105. 中 间体 105 在 6-OST 和 3-OST 催化下分别将 GlcN 单元的 C-6-位羟基硫酸化, 并选择性的将与 $L$-IdoA 相连的非还 原端 $\mathrm{GlcN}$ 的 C-3-位羟基硫酸化. 这样刘健和 Linhardt 等运用 8 个酶, 通过 11 步反应以 $42 \%$ 的总收率得到了 一个与二糖相连的肝素戊糖抗凝片段 107.

通过改变的酶的使用次序, 刘健和 Linhardt 等运用 与上述相同的 8 个酶通过 13 步反应以 $34 \%$ 的收率合成 了另外一个与二糖相连的肝素戊糖序列 108 (Scheme 20).

通过化学酶法合成策略得到的上述两个肝素类寡 糖片段在体内和体外活性评价中都表现出与化学合成 得到的磺达肝素(Fondaparinux)相似的抗凝活性. 这一 首次实现的肝素类寡糖化学酶法合成途径具有极大的 灵活性, 有望应用于一些特定肝素寡糖序列的合成. 由 于合成中涉及的酶较多(针对目前的序列需要 8 种), 合 成中需要的砌块为价格昂贵的核苷活化的糖基给体
(UDP-GlcNTFA，UDP-GlcNAc 和 UDP-GlcUA)，这些不 利因素使得化学酶法合成策略真正应用于肝素类寡糖 的大量合成还面临巨大的挑战.

\section{3 结束语}

肝素因其结构复杂性和多重生物活性在今后一个 时期将会继续成为糖化学和糖生物学研究一个热点领 域. 这方面的研究进展将会为开发新的抗凝药物, 以及 肝素类抗炎、抗病毒、抗肿瘤药物提供坚实的基础. 近 年来发展的模块式合成和 “一锅法” 反应使得肝素类寡 糖的化学合成取得了长足进展, 但目前的策略仍然是特 定序列导向的定向合成. 正如 Paulsen ${ }^{[44]}$ 在 20 世纪 80 年代指出的那样, 葟糖化学合成没有一个通用的方 法，而我们目前仍面临着这样一个相同的问题，这就 需要发展新的寡糖合成方法和策略来满足针对不同肝 素序列的快速合成. 化学酶法合成在肝素类寡糖合成上 的首次实现为肝素类寡糖的合成提供了一个新的途径, 这一策略使得在实验室规模上快速高效的构建一系列 肝素寊糖化合物库, 进而深入研究肝素的构效关系 (SAR)成为可能. 然而, 化学酶法合成途径在肝素类塞 糖的大量合成上还需要解决酶的来源以及糖基给体的 大量合成所面临的挑战.

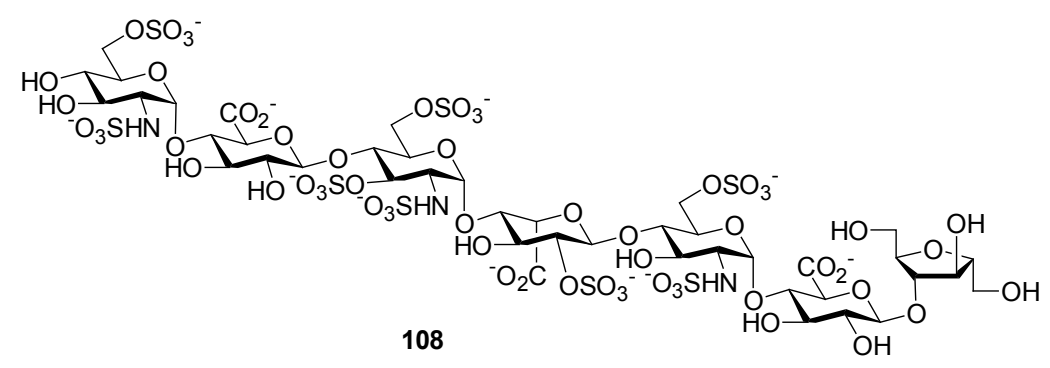

Scheme 20

\section{References}

[1] Casu, B.; Lindahl, U. Adv. Carbohydr. Chem. Biochem. 2001, 57, 159.

[2] Capila, I.; Linhardt, R. J. Angew. Chem., Int. Ed. 2002, 41, 391.

[3] Raman, R.; Sasisekharan, V.; Sasisekharan, R. Chem. Biol. 2005, $12,267$.

[4] Sasisekharan, R.; Raman, R.; Prabhakar, V. Ann. Rev. Biomed. Eng 2006, 8, 181.

[5] Petitou, M.; Duchaussoy, P.; Lederman, I.; Choay, J.; Sinay, P.; Jacquinet, J. C.; Torri, G. Carbohydr. Res. 1986, 147, 221.

[6] Petitou, M.; Duchaussoy, P.; Lederman, I.; Choay, J.; Jacquinet, J. C.; Sinay, P.; Torri, G. Carbohydr. Res. 1987, 167, 67.

[7] Petitou, M.; van Boeckel, C. A. A. Angew. Chem., Int. Ed. 2004, 43, 3118.

[8] Kovensky, J. Curr. Med. Chem. 2009, 16, 2338.

[9] Gandhi, N. S.; Mancera, R. L. Drug Discovery Today 2010, 15, 1058 .

[10] Poletti, L.; Lay, L. Eur. J. Org. Chem. 2003, 2999.
[11] Karst, N. A.; Linhardt, R. J. Curr. Med. Chem. 2003, 10, 1993.

[12] Whitelock, J. M.; Iozzo, R. V. Chem. Rev. 2005, 105, 2745.

[13] Orgueira, H. A.; Bartolozzi, A.; Schell, P.; Seeberger, P. H. Angew. Chem., Int. Ed. 2002, 41, 2128.

[14] Orgueira, H. A.; Bartolozzi, A.; Schell, P.; Litjens, R.; Palmacci, E. R.; Seeberger, P. H. Chem. Eur. J. 2003, 9, 140.

[15] Lohman, G. J. S.; Seeberger, P. H. J. Org. Chem. 2004, 69, 4081.

[16] Adibekian, A.; Bindschadler, P.; Timmer, M. S. M.; Noti, C.; Schutzenmeister, N.; Seeberger, P. H. Chem. Eur. J. 2007, 13, 4510.

[17] Prabhu, A.; Venot, A.; Boons, G. J. Org. Lett. 2003, 5, 4975.

[18] Arungundram, S.; Al-Mafraji, K.; Asong, J.; Leach, F. E.; Amster, I. J.; Venot, A.; Turnbull, J. E.; Boons, G. J. J. Am. Chem. Soc. 2009, 131, 17394.

[19] Cao, H. Z.; Yu, B. Tetrahedron Lett. 2005, 46, 4337.

[20] Zhou, Y.; Lin, F.; Chen, J.; Yu, B. Carbohydr. Res. 2006, 341, 1619.

[21] Chen, J.; Zhou, Y.; Chen, C.; Xu, W.; Yu, B. Carbohydr. Res. 2008, 343, 2853.

[22] Chen, J. F.; Yu, B. Tetrahedron Lett. 2008, 49, 1682. 
[23] Chen, C.; Yu, B. Bioorg. Med. Chem. Lett. 2009, 19, 3875.

[24] Hung, S. C.; Thopate, S. R.; Chi, F. C.; Chang, S. W.; Lee, J. C.; Wang, C. C.; Wen, Y. S. J. Am. Chem. Soc. 2001, 123, 3153.

[25] Lee, J. C.; Lu, X. A.; Kulkarni, S. S.; Wen, Y. S.; Hung, S. C. J. Am. Chem. Soc. 2004, 126, 476.

[26] Lu, N.-D. L.; Shie, C.-R.; Kulkarni, S. S.; Pan, G.-R.; Lu, X.-A.; Hung, S.-C. Org. Lett. 2006, 8, 5995.

[27] Hu, Y. P.; Lin, S. Y.; Huang, C. Y.; Zulueta, M. M. L.; Liu, J. Y.; Chang, W.; Hung, S. C. Nat. Chem. 2011, 3, 557.

[28] Wang, C.-C.; Lee, J.-C.; Luo, S.-Y.; Kulkarni, S. S.; Huang, Y.-W.; Lee, C.-C.; Chang, K.-L.; Hung, S.-C. Nature 2007, 446, 896.

[29] Codee, J. D. C.; Stubba, B.; Schiattarella, M.; Overkleeft, H. S.; van Boeckel, C. A. A.; van Boom, J. H.; van der Marel, G. A. J. Am. Chem. Soc. 2005, 127, 3767.

[30] Zhang, Z. Y.; Ollmann, I. R.; Ye, X. S.; Wischnat, R.; Baasov, T.; Wong, C. H. J. Am. Chem. Soc. 1999, 121, 734.

[31] Polat, T.; Wong, C. H. J. Am. Chem. Soc. 2007, 129, 12795.

[32] Yu, H. N.; Furukawa, J.; Ikeda, T.; Wong, C. H. Org. Lett. 2004, 6, 723.

[33] Huang, X. F.; Huang, L. J.; Wang, H. S.; Ye, X. S. Angew. Chem., Int. Ed. 2004, 43, 5221.
[34] Wang, Z.; Xu, Y. M.; Yang, B.; Tiruchinapally, G.; Sun, B.; Liu, R. P.; Dulaney, S.; Liu, J. A.; Huang, X. F. Chem. Eur. J. 2010, 16, 8365.

[35] Al-Horani, R. A.; Desai, U. R. Tetrahedron 2010, 66, 2907.

[36] Miller, S. C. J. Org. Chem. 2010, 75, 4632.

[37] Ingram, L. J.; Taylor, S. D. Angew. Chem., Int. Ed. 2006, 45, 3503.

[38] Desoky, A. Y.; Taylor, S. D. J. Org. Chem. 2009, 74, 9406.

[39] Tiruchinapally, G.; Yin, Z.; El-Dakdouki, M.; Wang, Z.; Huang, X. Chem. Eur. J. 2011, 17, 10106.

[40] Chen, J. H.; Avci, F. Y.; Munoz, E. M.; McDowell, L. M.; Chen, M.; Pedersen, L. C.; Zhang, L. J.; Linhardt, R. J.; Liu, J. J. Biol. Chem. 2005, 280, 42817.

[41] Martin, J. G.; Gupta, M.; Xu, Y.; Akella, S.; Liu, J.; Dordick, J. S.; Linhardt, R. J. J. Am. Chem. Soc. 2009, 131, 11041.

[42] Liu, R. P.; Xu, Y. M.; Chen, M. A.; Weiwer, M.; Zhou, X. X.; Bridges, A. S.; DeAngelis, P. L.; Zhang, Q. S.; Linhardt, R. J.; Liu, J. A. J. Biol. Chem. 2010, 285, 34240.

[43] Xu, Y.; Masuko, S.; Takieddin, M.; Xu, H.; Liu, R.; Jing, J.; Mousa, S. A.; Linhardt, R. J.; Liu, J. Science 2011, 334, 498.

[44] Paulsen, H. Angew. Chem., Int. Ed. 1982, 21, 155. 